\title{
Automatic 3-D Facial Fitting Technique for a Second Life Avatar
}

\author{
Hiroshi Dohi ${ }^{1}$ and Mitsuru Ishizuka ${ }^{2}$ \\ ${ }^{1}$ Dept. Information and Communication Engineering, Graduate School of \\ Information Science and Technology, University of Tokyo \\ ${ }^{2}$ Dept. Creative Informatics, Graduate School of Information Science and Technology, \\ University of Tokyo \\ 7-3-1, Hongo, Bunkyo-ku, Tokyo 113-8656, Japan \\ dohi@mi.ci.i.u-tokyo.ac.jp
}

\begin{abstract}
This paper describes an automatic 3-D facial fitting technique for a Second Life avatar. It is often difficult to create an original avatar by yourself that resembles a real person. In Second Life, the combinations of systemdefined parameters deform the shape of the avatar, and we can't control each vertex directly. It needs to encode the deformation information into many parameters. As a reference target, we make use of MRI data that scans the real face. It is stable and not affected by lighting and diffused reflection. In our experiments, we picked up 428 vertices on the base model for facial fitting. Using the iteration technique, more than $50 \%$ of vertices are just on the reference targets, and more than $85 \%$ are within $+/-3 \mathrm{~mm}$ of errors.
\end{abstract}

\section{Introduction}

A 3-D virtual environment is expected as one of post-WWW media interface. Many users share the 3-D world, and interact with each other through their avatars.

The avatar is a digital persona in a 3-D virtual world. There are two types of avatars. One is the avatar that has alternate identity and another is the avatar that resembles a real character. The former is the "anonymous" avatar. It can hide real name and protect user's privacy in real life. And the latter, "identity" may have special meaning to others. The avatar is associated with the person in real life.

It has been discussed for a long time about the avatar that resembles a famous character and "uncanny valley" hypothesis. The realistic face, of course, is not necessarily critical for avatar communication. However, in real life the famous character is often used for advertising, e.g. a movie star, a celebrity, an athlete, a politician, and so on.

The human has superior discrimination capability for human faces. It is not difficult for you to find your friend out of the crowd. Even the avatar that resembles your closest friend may have meaning in your local community group in the 3-D virtual world. 
It is often difficult to create an original avatar by yourself that resembles a real person.

For example, Linden Lab's Second Life [9] has gathered up more than 18 million registered users (called "residents") all over the world. One of salient features on Second Life is high-quality computer graphics. We can purchase various types of realistic objects, includes apparels, avatar accessories, and even avatar appearance, in Linden\$ currency at the marketplace in the Second Life world [10]. It is easy to become a different avatar. However it is difficult to create the avatar that resembles you by yourself, although Second Life offers an avatar appearance tool to all residents. The tool is simple to use, but it requires skills of both art and computer graphics for creating the fascinating avatar.

In this paper, we have empirical study of automatic 3-D facial fitting technique for a Second Life avatar. It needs to encode deformation information to many visual parameters.

\section{Avatar Appearance in Second Life}

In Second Life, many avatars live in the 3-D virtual environment. Each user can watch the world from arbitrary viewpoint.

Table 1. Shape parameters of the appearance tool in Second Life

\begin{tabular}{|c|c|c|}
\hline Sub categories & & Parameters \\
\hline Body* & 3 & Height, Body Thickness, Body Fat \\
\hline Head & 11 & $\begin{array}{l}\text { Head Size*, Head Stretch, Head Shape, Egg Head, Head } \\
\text { Length, Face Shear*, Forehead Angle, Brow Size, Upper } \\
\text { Cheeks, Lower Cheeks, Cheek Bones }\end{array}$ \\
\hline Eyes & 11 & $\begin{array}{l}\text { Eye Size, Eye Opening, Eye Spacing, Outer Eye Corner, } \\
\text { Inner Eye Corner, Eye Depth, Upper Eyelid Fold, Eye } \\
\text { Bags, Puffy Eyelids, Eyelash Length*, Eye Pop* }\end{array}$ \\
\hline Ears* & 4 & Ear Size, Ear Angle, Attached Earlobes, Ear Tips \\
\hline Nose & 11 & $\begin{array}{l}\text { Nose Size, Nose Width, Nostril Width, Nostril Division, } \\
\text { Nose Thickness, Upper Bridge, Lower Bridge, Bridge } \\
\text { Width, Nose Tip Angle, Nose Tip Shape, Crooked Nose* }\end{array}$ \\
\hline Mouth & 9 & $\begin{array}{l}\text { Lip Width, Lip Fullness, Lip Thickness, Lip Ratio, Mouth } \\
\text { Position, Mouth Corner, Lip Cleft Depth, Lip Cleft, Shift } \\
\text { Mouth* }\end{array}$ \\
\hline Chin & 9 & $\begin{array}{l}\text { Chin Angle, Jaw Shape, Chin Depth, Jaw Angle, Jaw Jut, } \\
\text { Jowls, Chin Cleft, Upper Chin Cleft, Chin-Neck }\end{array}$ \\
\hline Torso* & 12 & $\begin{array}{l}\text { Torso Muscles, Neck Thickness, Neck Length, Shoulders, } \\
\text { Breast Size, Breast Buoyancy, Breast Cleavage, Arm Length, } \\
\text { Hand Size, Torso Length, Love Handles, Belly Size }\end{array}$ \\
\hline Legs* & 8 & $\begin{array}{l}\text { Leg Muscles, Leg Length, Hip Width, Hip Length, Butt Size, } \\
\text { Saddle Bags, Knee Angle, Foot Size }\end{array}$ \\
\hline
\end{tabular}

* Not evaluated in our experiments for facial fittings. 
The Second Life system uses a server-client model. The client (viewer) has the base 3-D wire frame model of the avatar. The position of each vertex is not transferred. Instead, the server distributes packets for visual parameters to all clients. It defines 218 visual parameters.

The Second Life official viewer includes some built-in tools. An avatar appearance tool is designed for modifying face / body shapes and creating clothes.

In the avatar appearance tool, "Body Parts" consists of 4 categories, i.e., Shape, Skin, Hair, and Eyes. The Shape category has 9 sub categories.

Table 1 shows shape parameters of the appearance tool.

The appearance tool can control various parameters listed on Table 1 by manipulating some slide bars. It is easy to create your original avatar.

However, it is more difficult to create the avatar that resembles a real person than you thought. Various techniques have been proposed for 3-D facial fitting. Blanz showed 3-D face reconstructions using a 3-D morphable model and optic flow algorithm $[1,2,8]$.

In Second Life, there are many constraints for avatar deformation.

- We can't control any vertices of the model directly.

- A single slide bar controls one or more visual parameters. It moves a group of vertices simultaneously. Each vertex moves in the straight line along with the slide bar value. Many vertices in the group move to different directions with different speeds in 3-D space.

- It is system-defined that which vertices are included in the group. The moving direction and speed of each vertex are also system-defined.

- The groups of vertices are overlapped. More than 40 slide bars may move a same vertex.

- There are about 50 slide bars for facial shape control. The range is usually " 0 to 100 " (or " -50 to +50 ") and it is restricted to be integer values. As a result, it can create more than $10^{100}\left(=101^{50}\right)$ facial shapes theoretically. It may include duplicated shapes.

The new position of the vertex $\boldsymbol{V}_{\boldsymbol{n}}$ is determined by following equations.

$$
\begin{aligned}
\vec{V}_{n} & =f\left(\vec{v}_{n}, p_{1}, \ldots, p_{m}\right) \\
& =\vec{v}_{n}+\sum_{i=1}^{m} w_{i}\left(p_{i}\right) \cdot \vec{d}_{i, n}
\end{aligned}
$$

$\boldsymbol{v}_{\boldsymbol{n}}$ is the base position of the vertex $n . \boldsymbol{p}$ is a morphing parameter and it is restricted to be integer values. $\boldsymbol{m}$ is the index of morphing parameters for facial control. $\boldsymbol{d}$ is a morphing vector. Each vertex has own vector on each morphing parameter. $\boldsymbol{w}_{i}\left(\boldsymbol{p}_{\boldsymbol{i}}\right)$ returns the weight (coefficient) of the morphing vector. Each morphing parameter has own range of $\boldsymbol{w}$. Both the range of $\boldsymbol{w}$ and the morphing vector are system-defined. 


\section{Facial Fitting Technique}

\subsection{MRI Data}

As the reference target for facial fitting, we make use of the MRI data that scans the real face. "MRI (Magnetic Resonance Imaging)" is a medical imaging technique to visualize detailed internal tissues of the human body.

MRI data has following features.

- It is non-invasive measure, and it is safe for human body since it doesn't use ionizing radiation unlike $\mathrm{X}$-rays technique.

- It can discriminate internal tissues. It can capture even the shape of the rear head covered with hair.

- It has no blind spots and no occlusion problem.

- MRI data is stable since it isn't affected by diffuse reflection.

A MRI system is very expensive and requires large space because it makes use of strong magnetic field. There are, however, unexpectedly many MRI units for medical use. According to the "OECD Health Data 2010," [7] there are 43.1 MRI units (and 97.3 computed tomography scanners) par million populations in Japan in 2008. And the number of MRI units increases twice from 2000 to 2008 across OECD (Organisation for Economic Co-operation and Development) membership countries.

The problem is that it is difficult to get raw image data from a medical institution. MRI data is one of medical data that includes private information, and then it is strictly controlled to bring it out.

\subsection{Basic Algorithm}

As the reference facial model, we used numerical human-body models developed by National Institute of Information and Communications Technology (NiCT), JAPAN [6]. These models are developed for the research on high-precision dosimetric

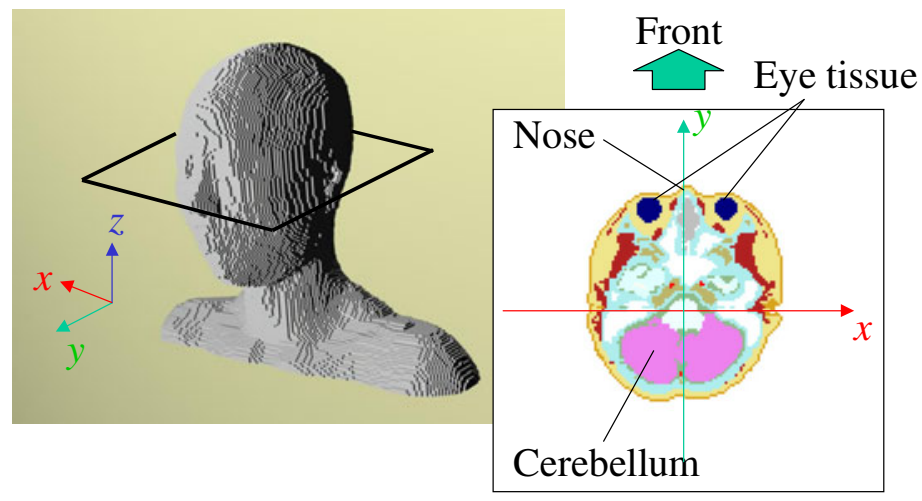

Fig. 1. A sliced MRI image 
technology. These are voxel (volumetric pixel) data based on accumulated MRI images. With post processing, internal tissues are already labeled. The spatial resolution is $2 \times 2 \times 2 \mathrm{~mm}$. The voxel size is $320 \times 160 \times 804$ for the female. It is inhibited to specify the test subjects under the license agreement.

Fig. 1 shows an example of the MRI image sliced on the $\boldsymbol{x}-\boldsymbol{y}$ plane.

We make fitting the base model of the avatar to the reference target by combinations of system-defined parameters.

Our problem is to determine a set of morphing parameter values $\left(\boldsymbol{p}_{\boldsymbol{0}}, \boldsymbol{p}_{1}, \ldots \boldsymbol{p}_{\boldsymbol{m}}\right)$ that achieve the lowest errors below. $\boldsymbol{p}$ is integer value (from 0 to 100), and the integer programming problem is much more difficult.

$$
\text { Errors }=\sum_{i=1}^{n}\left|V i_{y}-D\left(V i_{x}, V i_{z}\right)\right|
$$

$\boldsymbol{D}$ is depth information of the reference face. The set of parameter values $\left(\boldsymbol{p}_{\boldsymbol{0}}, \boldsymbol{p}_{\boldsymbol{1}}, \ldots\right.$ $\boldsymbol{p}_{\boldsymbol{m}}$ ) moves $\boldsymbol{v}_{\boldsymbol{i}}$ to the new position $\boldsymbol{V}_{\boldsymbol{i}}(\boldsymbol{x}, \boldsymbol{y}, \boldsymbol{z})$, and $\boldsymbol{D}$ depends on the position $(\boldsymbol{x}, \boldsymbol{z})$ of Vi.

In order to decide parameters $\left(\boldsymbol{p}_{0}, \boldsymbol{p}_{1}, \ldots \boldsymbol{p}_{\boldsymbol{m}}\right)$, we adopted a simple hill-climbing algorithm.

1. Choose one of morphing parameters $\boldsymbol{p}$ in random order.

2. Change the parameter value from 0 to 100 , and calculate each error value.

3 . If error value is lower, update the parameter value.

4. Iterate above steps until all parameters are not changed.

\subsection{Optimization}

The basic algorithm above needs to check parameters over and over again, since other parameters may move the same vertex.

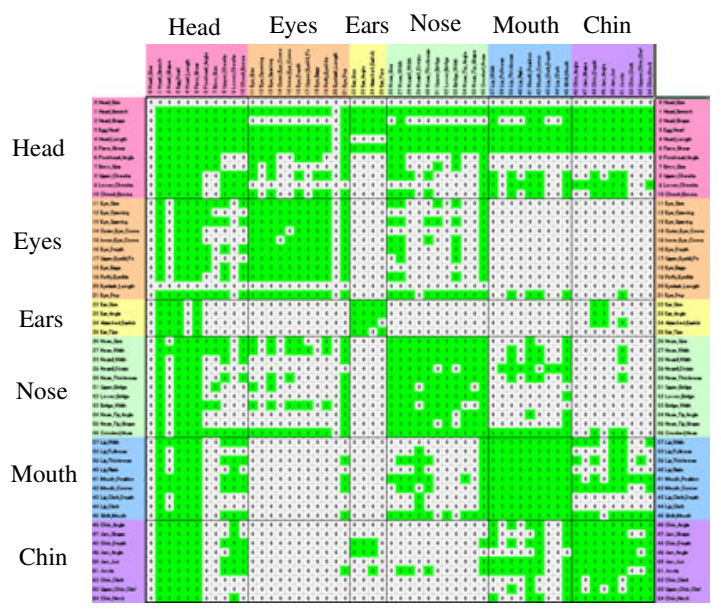

Fig. 2. Dependency matrix for morphing parameters 
In order to decrease redundancy of the basic algorithm, we analyzed dependency relation of morphing parameters from avatar definition files, and made a dependency matrix

Fig. 2 shows the dependency matrix for morphing parameters.

The row of the matrix is the parameter that moves some vertices, and the column is the affected parameters. It is the symmetric matrix.

We use a bit vector in order to manage parameters efficiently. "The bit is 1" means that when we control the corresponding parameter it may make error value lower. The initial state of the bit vector is all 1 . We choose one of the parameter that the bit is 1. Assume the current bit vector is "00110011." $\left(\boldsymbol{b}_{2}=\boldsymbol{b}_{3}=\boldsymbol{b}_{6}=\boldsymbol{b}_{7}=1\right)$ It means we have 4 candidates, i.e., parameters $\boldsymbol{P}_{2}, \boldsymbol{P}_{3}, \boldsymbol{P}_{6}$, and $\boldsymbol{P}_{7}$. For example, we choose $\boldsymbol{P}_{3}$, and clear the bit $\left(\boldsymbol{b}_{3}=0\right)$. Then we change the $\boldsymbol{P}_{3}$ value from 0 to 100 , and calculate each error value. If error value is larger or same, the $\boldsymbol{P}_{\boldsymbol{3}}$ value is unchanged and the bit vector shows next candidates. If lower, the $\boldsymbol{P}_{\mathbf{3}}$ value is updated. Then it adds the dependency bit vector (the column line for $\boldsymbol{P}_{3}$ of the dependency matrix.) The column line "01101000" for $\boldsymbol{P}_{\mathbf{3}}$ means that $\boldsymbol{P}_{\mathbf{3}}$ affects $\boldsymbol{P}_{\boldsymbol{1}}, \boldsymbol{P}_{\mathbf{2}}$, and $\boldsymbol{P}_{\mathbf{4}}$. When the bit vector is all 0 after iterations, we get the set of parameters that error value is one of local minima.

In order to escape local minima, it replaces some parameter values with random ones, and adds the dependency bit vector for the parameter to the current bit vector. Then it iterates above steps until the current bit vector becomes all 0 .

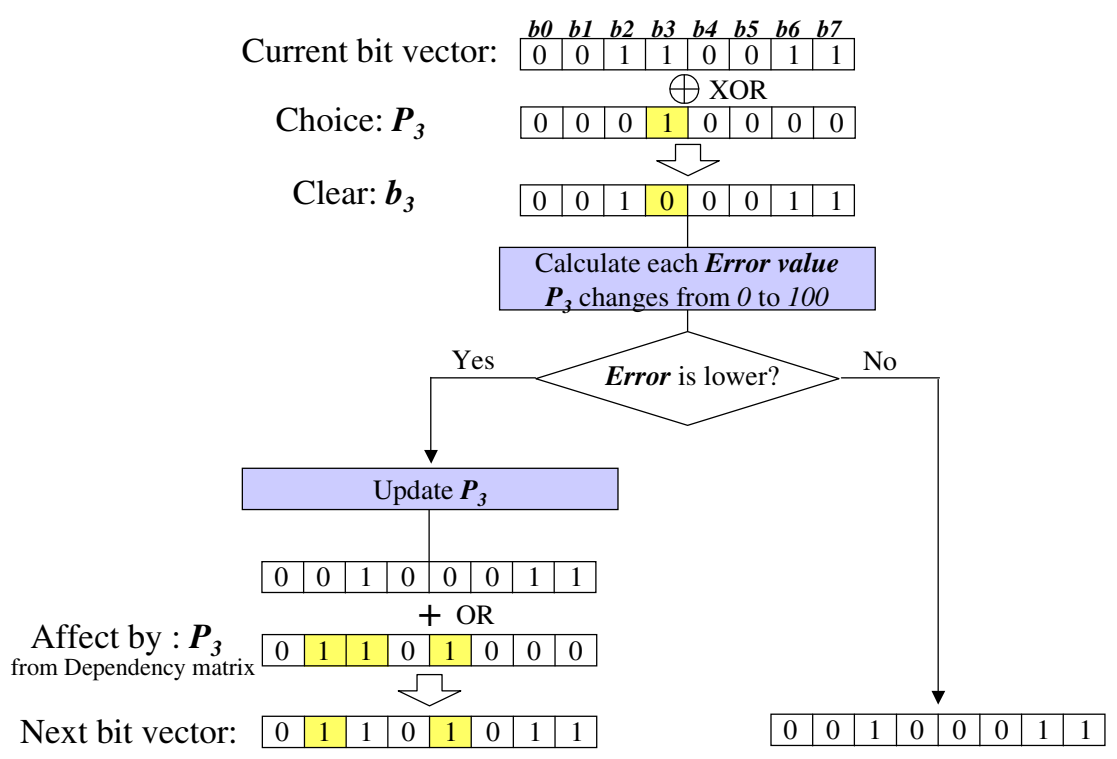

Fig. 3. Optimization of the basic hill-climbing algorithm 


\section{Results and Discussion}

\subsection{Facial Modeling Using MRI Data}

It is useful to make use of MRI image. MRI has strong relation with various visualization techniques on computer graphics field, e.g. 3-D image reconstruction, voxel rendering, etc. However its data in itself has received little attention so far for facial modeling and animation, although MRI can scan a human body in detail.

Fig. 4 shows an example of the 3-D image reconstructed from MRI data.

The shape of the face is extracted well, even the rear head. The remarkable feature is that MRI data is stable. It is easy to extract outlines from the MRI voxel data.

It may seem that the shape is a coarse grid at a glance, but it is negligible since the size of the unit cube is small, $2 \times 2 \times 2(\mathrm{~mm})$ in our experiments.

3-D digitizers with laser (or optical) scan have been used for facial modeling. The 3-D digitizer generates a surface model, and the resolution is generally more accurate $(<1 \mathrm{~mm})$ than that of MRI. However, it can't capture hair data because strands often diffuse and reflect sensor beam. Other data, e.g., eyeball, eyebrow, beard and mustache, etc. are sometimes missing or wrong. It may require post processing for data modification manually.

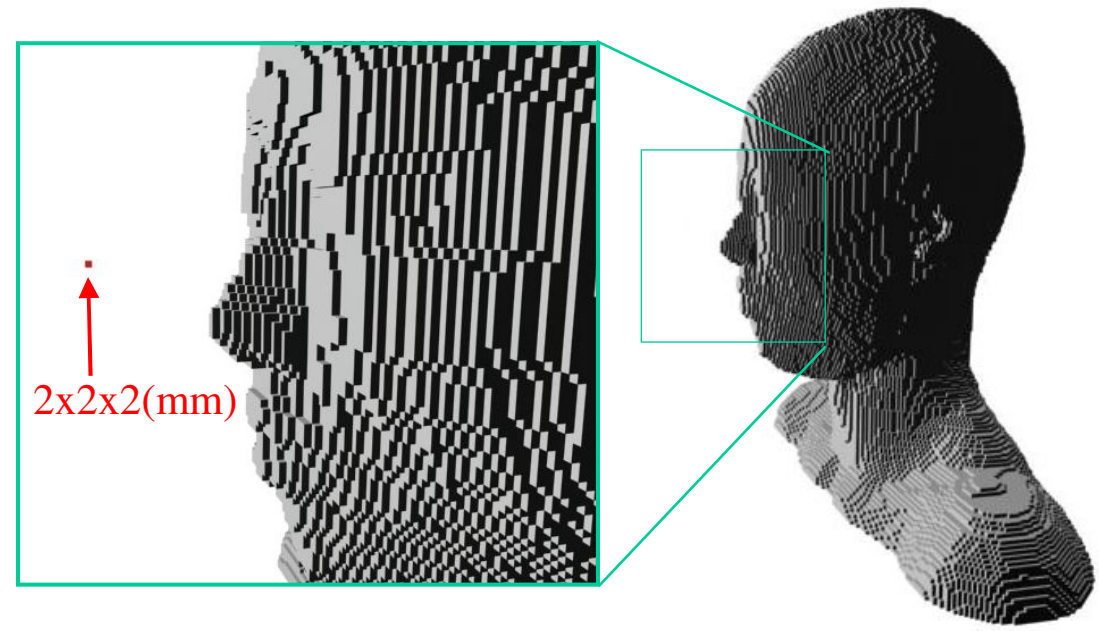

Fig. 4. 3-D image reconstructed from MRI data

\subsection{3-D Facial Fitting for Second Life Avatar}

Occluded (or overlapped) vertices (covered by other surface) on the base facial model may cause improper results, because our experiments use depth information. We removed excess vertices that could cause problems, e.g. contours of the side face, around of eyes, ears, nostril, mouth / lip, and rear head. The head part of the base 
model is composed of 1132 vertices (without LOD vertices), and we picked up 428 vertices for our evaluation.

In our experiments, we don't make fitting the rear head because Second Life has only a few parameters for deforming it. The rear head is often covered with hair. Hair with various hairstyles is another item.

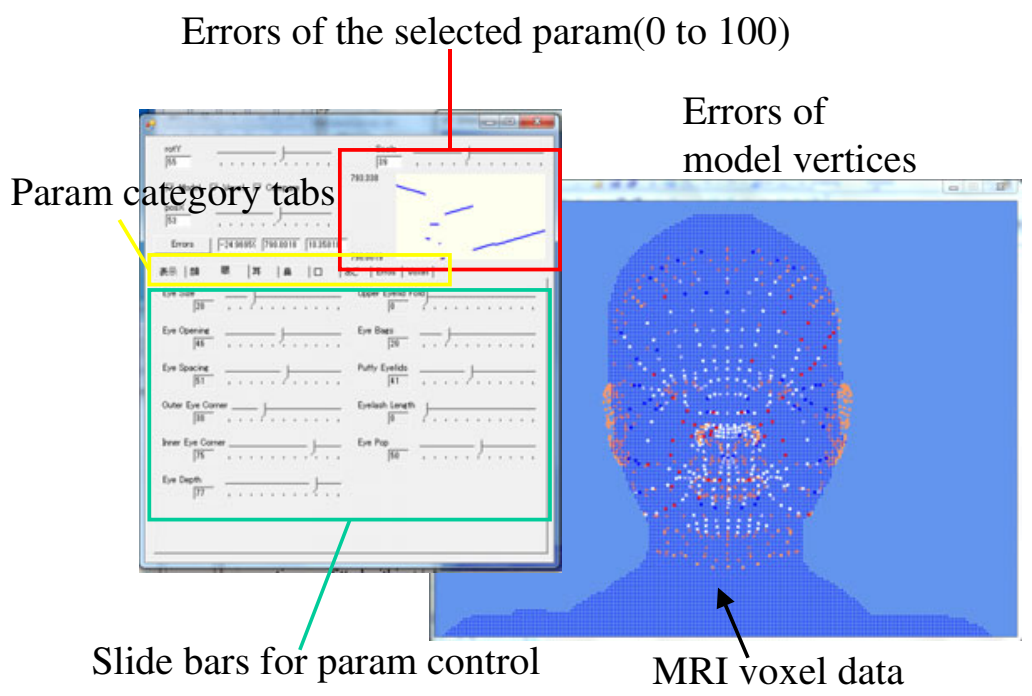

Fig. 5. A screen shot of our evaluation system

Fig. 5 shows a screen shot of our evaluation system. There are two windows. One is the parameter controller window (left), and another is the error display window (right).

The parameter controller has parameter category tabs and many slide bars. These have same functions as the avatar appearance tool on the Second Life viewer.

In our system, when we click on the label of the slide bar, it calculates all error values that the selected parameter changes from 0 to 100 automatically. Then, it displays those results as a graph at the upper right of the window, and the slide bar is set to the parameter value that gives the lowest error value.

It also has the function that iterates until all parameters become unchanged. Finally, we can get the set of parameter values.

The error display shows the position and depth error of each vertex.

The shape of head (small blue dots) is MRI voxel data. White boxes show model vertices that are just fitting. Red boxes have plus errors, and blue boxes have minus errors. Yellow boxes are model vertices that not evaluated in our experiments.

Fig. 6 is the side view. It finds fitting is well (white boxes) along the outline from forehead to chin. On the other hand, the lower part of the rear head is apart from the reference model since there is no morphing parameter. These vertices are not evaluated (yellow boxes). 


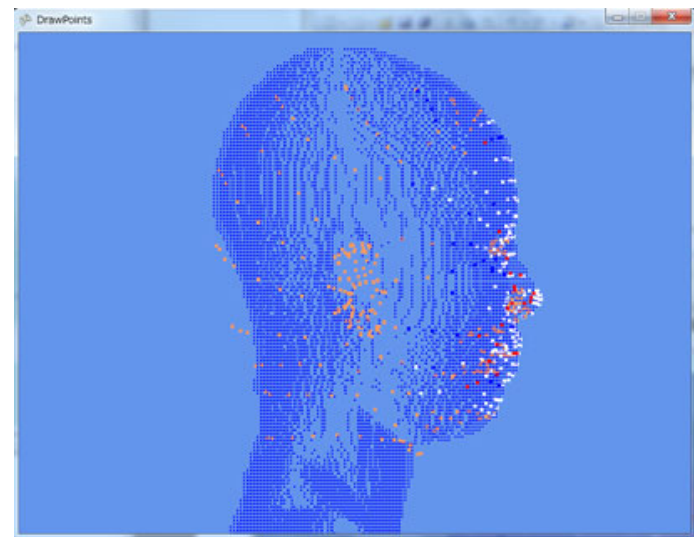

Fig. 6. Fitting results (side view)

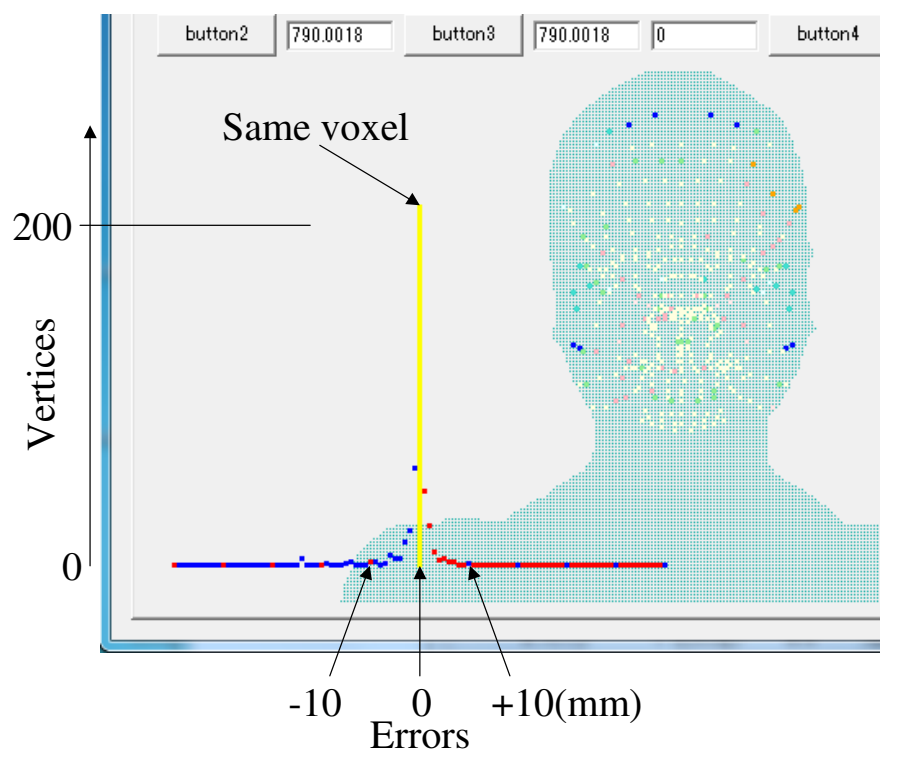

Fig. 7. A histogram for fitting results

Fig. 7 shows a histogram for fitting results.

After some iteration for escaping local minima, the score of total errors for 428 vertices is under 750. (This score has no unit. However in our experiments, the unit of the score is equal to "mm".) More than $50 \%$ of vertices are just on the reference targets (same voxel). More than $85 \%$ of vertices are fitted within $+/-3 \mathrm{~mm}$ of errors (neighbor voxel), and $95 \%$ are within $+/-5 \mathrm{~mm}$. It is good results, and we can find leaner silhouette of the face. It is difficult to achieve the score by manual fitting operation with the error display. 
The lowest errors value is not necessarily bring the best fitting result. We don't use "asymmetric" parameters for our evaluation like "Face Shear", "Eye Pop", "Crooked Nose", and "Shift Mouth" listed on Table 1. Although the use of these parameters may return the lowest errors value, it often brings an unnatural face with parameter settings that the human never choose.

\section{Conclusion}

In this paper we have described the empirical study of automatic facial fitting for Second Life avatar.

We used MRI data as our reference model. It is useful for facial modeling. It will be helpful for computer animation field if it becomes easier to make use of raw image data measured by MRI for medical use.

After the iterations, more than $50 \%$ of vertices are just on the reference targets, and more than $85 \%$ are within $+/-3 \mathrm{~mm}$ of errors. It is better than that of manual fitting operation with the error display.

Both MRI data and textures will improve the accuracy of facial fitting.

\section{References}

1. Blanz, V., Vetter, T.: A Morphable Model for the Synthesis of 3D Faces. In: Proc. of ACM SIGGRAPH 1999, pp. 187-194 (1999)

2. Blanz, V., Vetter, T.: Face Recognition Based on Fitting a 3D Morphable Model. IEEE Trans. On Pattern Analysis and Machine Intelligence 25(9), 1063-1074 (2003)

3. Dohi, H., Ishizuka, M.: A Life-like Agent Interface System with Second Life Avatars on the OpenSimulator Server. In: Proc. of 13th Int'l Conf. on Human-Computer Interaction, HCII 2009 (2009)

4. Koda, T., Maes, P.: Agents with Faces: The Effects of Personification of Agents. In: Proc. HCI 1996, pp. 98-103. The British HCI Group (1996)

5. Maes, P.: Agents that Reduce Work and Information Overload. Commu. ACM 37(7), 3140 (1994)

6. Nagaoka, T., Watanabe, S., Sakurai, K., Kunieda, E., Watanabe, S., Taki, M., Yamanaka, Y.: Development of Realistic High-Resolution Whole-Body Voxel Models of Japanese Adult Male and Female of Average Height and Weight, and Application of Models to Radio-Frequency Electromagnetic-Field Dosimetry. Physics in Medicine and Biology 49, $1-15$ (2004)

7. OECD Health Data 2010 - Version (October 2010), http: / / www . oecd. org/

8. Patel, A., Smith, W.A.P.: 3D Morphable Face Models Rivisited. In: Proc. of Int'l Conf. On Computer Vision and Pattern Recognition, CVPR 2009, pp. 1327-1334 (2009)

9. Second Life official site, http: / / secondlife.com/

10. Second Life Marketplace, https : / marketplace.secondlife.com/ 